\title{
Studying the Navigation Assistance System for the Visually Impaired and Blind Persons and ICT use by their Caretakers
}

\author{
Babar Chaudary, Iikka Paajala \\ University of Oulu, OASIS Research \\ Unit. Oulu, Finland \\ babar.chaudary, iikka.paajala@oulu.fi
}

\author{
Leena Arhippainen \\ University of Oulu, INTERACT \\ Research Unit. Oulu, Finland \\ leena.arhippainen@oulu.fi
}

\author{
Petri Pulli \\ University of Oulu, OASIS Research \\ Unit. Oulu, Finland \\ petri.pulli@oulu.fi
}

\begin{abstract}
Telecare solutions have been seen as a potential means of addressing the future care needs of ageing societies. With the growing proportion of dependent people (ageing, disabled users), tele-assistance and tele-monitoring platforms will play a significant role to provide an efficient and less-costly remote care and support. It will allow aged and disabled persons to maintain their independence and lessen the burden and cost of caregiving. In the case of visually impaired (VIP) and blind persons, guide dog and white cane provide them a fair degree of independence. However, those are very limited in guiding the user towards a specific desired location, especially in an unknown environment. The assistance of other people presents a feasible solution, though it does not improve the idea of autonomous guidance and privacy. This paper presents a tele-guidance based navigation assistance system that is based on the idea that a blind or VIP pedestrian can be assisted by haptics based cues and spoken instructions from a remote caretaker who receives a live video stream from a camera carried by the blind or VIP. Also, we present results of the study conducted in Pakistan with 14 blind and VIP participants for testing haptics user interface concept of proposed teleguidance system. In this study also two caretakers are interviewed. In addition, we present results of the attitude towards assistive technology study conducted with 19 blind and VIP in Finland. Moreover, we introduce the environmental model to aid development of assistive tools for blind and VIP.
\end{abstract}

\section{INTRODUCTION}

Vision loss is a serious impairment that deprives a human of approximately $80-90 \%$ perceptual abilities and has a detrimental effect on professional, social, and personal quality of life of a person. People who suffer from visual impairments face major challenges to their daily lives, as they suffer from adverse effects to their performance in many situations [1].

It is estimated that globally there were 285 million VIP in the world, 39 million blind and 246 million having low vision in 2010 [2], [3]. According to estimations, 36 million people were blind, 217 million had moderate or severe vision impairment, and 188 million had mild vision impairment in 2015 [4]. The number of VIP are expected to grow. According to projections, 114.6 million people (of a total global population of 9.69 billion) would be blind and 587.6 million people would suffer moderate and severe vision impairment in 2050. It is estimated that 1.09 billion people aged 35 years and older and 666.7 million people aged 50 years and older are influenced by near vision impairment due to uncorrected presbyopia [4], [5]. $65 \%$ of VIP and $82 \%$ of blind are 50 years and older [2], [3]. The growing number of elderly people results in increased problems caused by the old age such are chronic eye diseases [2].

Being blind or VIP does not mean losing the independence of getting to and from places whenever one wanted. People with no vision or limited vision can travel independently daily with their means best suited for them. Though one of the biggest challenges to independence for people who are visually impaired is associated with safe and efficient navigation [6-8]. For that purpose, people who are blind or visually impaired started using conventional navigation aids such as white canes, guide dogs, assistance by a guide (professional or family member, friend) or volunteer since long time back. With the technological advancement, assistive tools were also being developed using technology [9], [10]. Later, such tools were being referred as Assistive Technology cumulatively [11]. The term assistive technology encompasses technologies, equipment, devices, apparatus, services, systems, processes, and environmental modifications. Though studies reported, assistive tools have acceptance issues by blind and VIP. The threshold for accepting and starting the uses of assistive tools have number of potential reasons related to either assistive tools or user [12-14]. Tools-related reasons include accessibility, usability, safety, and appearance of the tool [15]. Whereas user-related reasons include user's previous experiences and attitude towards technology, technological prowess, and perceived need for the tool. Also, if the assistive tool marks the user as different may cause rejection [15], [16].

In this paper, we propose a teleguidance-based navigation support system for VIP and blind persons. The concept is based on the idea where a blind pedestrian is assisted by haptics based directional cues through pair of vibrators installed on grip of her smart cane and through spoken instructions from a remote caretaker who receives a live video stream from an IP camera carried by the VIP or blind person. The paper reports results of usability study conducted in Pakistan with 14 VIP or blind test subjects using smart grip-based haptics cues for navigation guidance concept to walk on a test trail. In this study, two caretakers were also interviewed. It is critical to investigate the willingness of the caretakers in such a collaborative assistance 
system as presented in this study, because it affects routine of both the subjects and their caretakers. The aim of the study was to investigate willingness and issues with acceptance of technological navigation aids by the VIP and use it as a guideline in developing and tailoring our proposed navigation aid for those. Therefore, we also present the qualitative study with 19 blind and VIP using a modified version of Unified Theory of Acceptance and Use of Technology (UTAUT2) to find out causes for acceptance issues in navigation tools for VIP. In addition, another aim of the study was to validate if UTAUT2-model is suitable for researching acceptance issues of navigation assistance tools of VIP.

\section{BACKGROUND}

Even in modern societies, common understanding of blindness and the needs of the VIP are poorly identified. The white cane and more rarely a guide dog are the primary mobility aids that are mainly associated with this disability. In spite of recent remarkable advancements in information and communication technologies (ICT) and electronics miniaturization, the devices that are termed as Electronic Travel Aids (ETA) are very slowly fighting their ways into the community of VIP. In fact, no single ETA has been widely accepted by the VIP and blind as a useful aid [2]. ETA is the general term encompassing a large class of assistive devices aiding the VIP in navigation and mobility. There has been a long lasting research record on ETAs helping the blind in obstacle avoidance and navigation. The idea of sensory substitution, i.e. replacing lack of stimuli from one sense by appropriate stimuli for another sense is the operating principle of all ETAs for the blind [2]. Following is a functional, rather than technological classification of these assistive devices [3]: 1. Obstacle Detectors, 2. Environment Imagers 3. Orientation \& Navigation Systems (ONSs).

The first two classes of aids are personal (wearable) devices that scan the environment in personal and near spaces. These devices have the task to assist blind people to intercept obstacles on their path. On the other hand, the third group of aids are the systems that offer sensing of far spaces and can acquire data from larger scale distributed networks, e.g. GPS, GIS, Digital Maps, and through wide spread access to the Internet and Wireless Communication Networks (e.g. RFIDs, Bluetooth, Wi-Fi, and GSM). An innovative class of ONSs is based on guiding the VIP by a remote human guide termed as tele-assistance/tele-guidance systems. The underpinning idea of this class of ETAs is that a blind pedestrian is guided by spoken instructions from a remote caretaker who remotely receives a video stream from a camera carried by the blind user. The remote vision facility permits the remote sighted guide to navigate the VIP user of the system in the immediate travel environment i.e. micro-navigation; e.g. the assistance in the avoidance of obstacles and other hazards in the path of travel, while the GPS and GIS data facilitates the navigation through the environment on a large scale i.e. macro-navigation.

The first reported system for remote guidance of the blind, was the system developed at the Brunel University, UK [4]. Three ICT technologies were combined to offer the teleassistance functionality; namely, GPS (Global Positioning
System), GIS (Geographic Information System) and video/voice transmission over the $3 \mathrm{G}$ mobile network. The system comprises of two units. The backpack mobile unit equipped with a portable camera and an audio headset that was carried by the blind user and a stationary PC based unit for remote caretaker. [17] developed a tele-assistance system using GPS, Digital maps, Bluetooth, and voice/video link was established over the GSM network within the High-Speed Downlink Packet Access (HSDPA). The system comprised of an ultra-mobile laptop computer worn in a shoulder bag, a digital webcam, and a GPS receiver attached to the shoulder strap, and a single-ear head-phone with a microphone and the assistant who remotely aided the VIP used any PC with a public IP address. In [17], the authors developed a remote guidance system where the visually impaired was equipped with a digital camera, a GPS receiver and a headset. Internet and GSM connections transmitted video/audio information and GPS data between the remote operator and the user. Using audio communication, the operator navigated the VIP towards a desired location and warned him about possible obstacles. Similar tele-assistance systems were developed by [19-21] for visually impaired pedestrians. In Japan, API AI Co [22] and BlindMaps [23] are developing technology and prototypes for making the white cane a connected device which can act as an interface to the urban environment and to the user's smartphone, has very many ideas that have potential to complement such system.

The former tele-guidance systems either used bulky back packs or special purpose mobile terminals to be carried by the VIP. This fact effected the acceptability of such systems by VIP at large. The advancement in computation capabilities of mobile devices and electronics miniaturization presented newer possibilities for developers to develop more user-friendly teleguidance systems for the VIP. The proposed tele-assistance based orientation and mobility system enables VIP to initiate a rich tele-guidance session with a remote caretaker when they need remote assistance during navigation. The remote caretaker can assist VIP remotely using voice commands and/or haptics/vibration based interfaces by using field of view of VIP and complementing data on his terminal.

\section{SYSTEM DESCRIPTION}

This chapter presents the proposed tele-guidance system, which comprises of two terminals, (1) VIP's terminal and (2) Remote caretaker's terminal (Fig. 1).

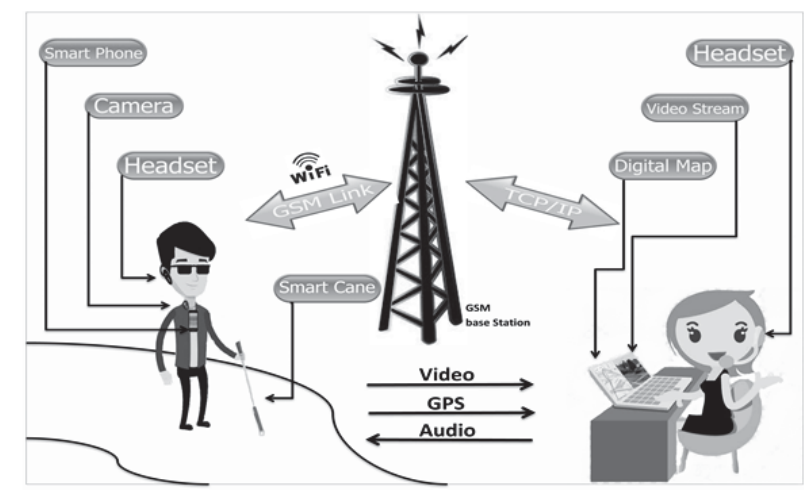

Fig. 1. Teleguidance based navigation assistance system scenario 


\section{A. VIP's terminal}

VIP's terminal comprises of four components:

1) Smart Cane (Braille Cell, Directional Vibrators, Bluetooth Interface): The smart cane contains directional vibrators for navigation assistance, tactile braille cell for tactile braille message, and Bluetooth interface to connect/control it with the smart phone.

2) Smart Phone: It provides connectivity with the remote caretaker's terminal through Wi-Fi or GSM based internet connection. The video, voice, and location coordinates of VIP using smart phone GPS are transmitted over the internet to remote caretaker. The readily available sensors e.g. GPS and network connectivity are used to provide remote caretaker with real time IoT data about VIP.

3) Bluetooth Webcam: A Bluetooth webcam connected to the smart phone through Bluetooth is mounted on the chest of the VIP. It sends real time video of the field of view of the VIP.

4) Bluetooth Headset (Open air Earpiece): The Bluetooth head set is used for voice communication.

The VIP's terminal will enable a blind or VIP to initiate a need based rich tele-guidance (audiovisual) assistance session with a remote caretaker. The remote caretaker will receive the call and an audio connection and location coordinates of blind or VIP position will be initialized when the remote assistance session is started once the caretaker accepts the call. If signals' quality is good, the caretaker will initialize the video channel to start video feed transmitted from VIP. The remote caretaker will now start providing on-site remote assistance to VIP remotely through haptics/vibration interfaces of smart cane accordingly and/or through voice commands accordingly. During the remote tele-guidance session, VIP will be able to mute the voice channel anytime while video still on if she wants to make sense of the environment by listening surrounding sounds. The remote caretaker will get a notification about it. VIPs will be able to configure more than one person as caretaker. If either of those higher in priority is not available, the help call will be automatically transferred to the one available. The selection of caretaker and hand over can also be based on need based support.

The smart cane is being developed as part of this project to provide caretaker with extra modalities i.e. voice and haptics to assist VIP remotely. As previous studies suggested and was also pointed by blind/VIP test subjects being interviewed in this study, engaging VIP's hearing during navigation activity is not recommended, haptics interface is critical especially for safe navigation. It's hazardous for the safety of the blind/VIP and chances are off missing some instruction in a noisy environment. The smart cane will be connected to the smart phone and will allow remote caretaker to assist VIP through vibration and haptics interfaces. This component will potentially extend the functionality of conventional voice-based teleguidance ONS systems as presented before. (Fig. 1)

\section{B. Remote Caretaker's Terminal}

The remote caretaker can either use a desktop, tablet, or phablet as a terminal based on his situation. It receives and initiates the VoIP video calls and renders VIP's real-time location coordinates. As VIP can configure many people as his caretaker, the system will help caretakers to mediate time and load of the assistance through availability status info. It will be studied if there is a need for the caretaker to override voice muting by VIP.

\section{Proposed Technologies}

The technologies proposed for different implementing features of the proposed tele-guidance system are:

1) Network Connectivity: Wi-Fi or GSM based internet connection will be used for making VOIP calls and sending real time GPS coordinates of VIP.

2) Unity 3D Game Engine: Unity3D platform is proposed for developing telepresence and guidance user interfaces to caretakers and VIP users. These technologies accelerates the development because of their advanced APIs that provide rich features especially making GUI for remote caretakers [24].

3) Encrypted Communication: To support privacy between VIP and remote caretaker, encrypted voice, video, and location data communication over the internet will be used. Linphone API that supports ZRTP protocol will be used to implement communication over the internet functionality [25].

4) ZRTP (composed of Z and Real-time Transport Protocol): ZRTP is a cryptographic key-agreement protocol to negotiate the keys for encryption between two end points in a Voice over Internet Protocol (VoIP) phone telephony call based on the Realtime Transport Protocol. It uses Diffie-Hellman key exchange and the Secure Real-time Transport Protocol (SRTP) for encryption. ZRTP stands for "Zimmermann Real-time Transport Protocol" and was developed by Silent Circle's own, Phil Zimmermann. ZRTP is key exchange protocol designed to enable VoIP devices to agree keys for encrypting media streams (voice or video) using SRTP [26]. The authors of ZRTP describe it as "Media Path Key Agreement for Secure RTP". This means that the ZRTP end points use the media stream rather than the signaling stream to establish the SRTP encryption keys. Many other key exchange protocols use the signaling stream (for example SIP or H.323) for media key exchange. The disadvantage of this approach is that the key exchange is visible to any intermediate device that processes the signaling stream. ZRTP's use of the media path for key agreement ensures that media keys are agreed directly between the caller and call recipient and those keys are not visible to any intermediate signaling device. This makes ZRTP an ideal choice for use on networks where signaling is processed by intermediate devices and where it is important to ensure call confidentiality [27].

\section{SMART GRIP TESTING STUDY WITH BLIND AND VIP PARTICIPANTS}

The aim of this study was to investigate both usability and acceptance issues relating to proposed navigation assistance system's VIP Terminal's haptics based user interface concept. It is important to understand how users experience the use of proposed technological navigation concept to design the experience of using the system as much assistive as possible. 


\section{A. Smart Grip - System description and design}

This prototype was developed specifically to collect end-user feedback about haptics UI of Smart Cane for teleguidance system. The test system comprised of two parts:

1. Smart Grip

2. Magnetic trail test bed

In our proposed teleguidance system described in section III, Smart Grip is one modular component of Smart Cane. In the tested system, it allows VIP or blind carrying Smart Cane to sense the vibration on their holding finger that is an acknowledgement for them navigating being oriented on the sidewalk testbed following test track. The magnetic trail testbed is made up of cardboard with a trail of magnets on it. That was made to test the Smart Grip UI. The concept of the magnetic trail is proposed already in an earlier study by the authors [28]. The Smart Grip tested in this study is developed in a way a modular component that works both on trail-based system [28] and with teleguidance system presented in this paper (Fig. 2).

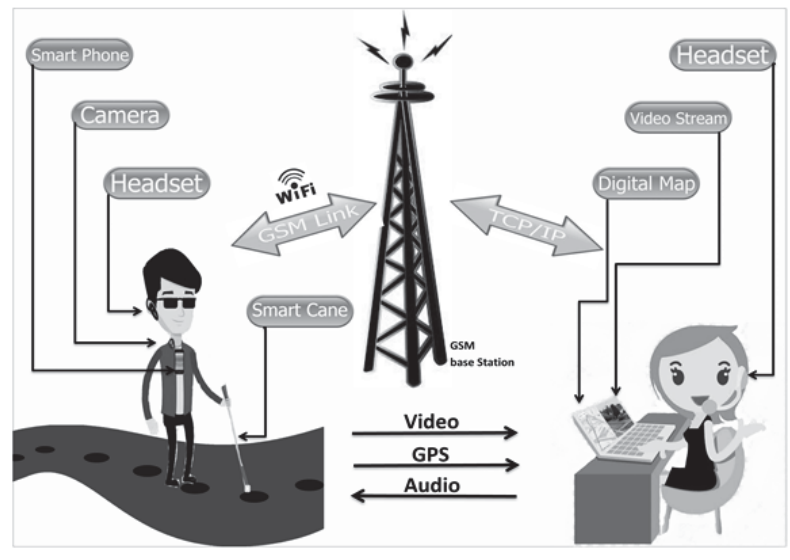

Fig. 2. Teleguidance based navigation assistance system with modular Smart Cane that works both with remote support and on a magnetic trail

The smart grip has three components (Fig. 3):

\section{Arduino Micro Controller}

2. Button type vibrator

3. Magnetic sensor

The magnetic sensor is installed at the tip of the white cane. The vibrator is installed at an optimum position at grip of the white cane so that it comes under pointing finger or thumb of the VIP or blind when holding the cane (Fig. 4). It makes using natural grip of the white cane to sense the vibration. Part of orientation training for VIP and blind includes holding the cane while keeping the pointing finger or thumb pointing towards the direction they are moving.

The magnetic trail sidewalk testbed is the second part of the prototype system. It is a model of sidewalk made of cardboard that has a magnetic trail. The magnetic trail is made using small circular shaped neodymium magnets. Each magnet is installed at 12 inches apart from preceding one (Fig. 5). As normal human step is about 30 inches, each step a VIP or blind detects two magnets.

\section{B. System usability test settings and participants}

The usability study was conducted to test the smart grip based haptics navigation assistance concept. The purpose of the study was to investigate whether such haptics-based navigation communication is usable in guiding VIP and blind. The objective of the study was to investigate:

- Can such haptics-based navigation assistance be usable for VIP and blind persons?

- What changes e.g. intensity of the vibration (and others) if they feel appropriate or want adjustments to make sensing the vibration more of a ease?

- Based on the one vibrator concept, what is their opinion about using more vibrators (2 or 3 ) to use those as directional and starting or stopping navigation aid (to be operated by remote caretaker).

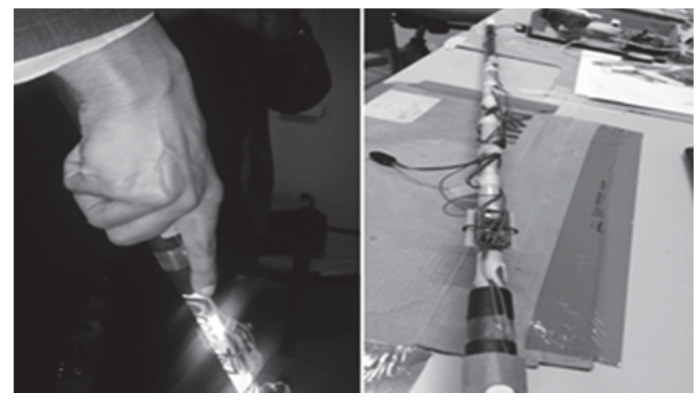

Fig. 3. Smart grip prototype

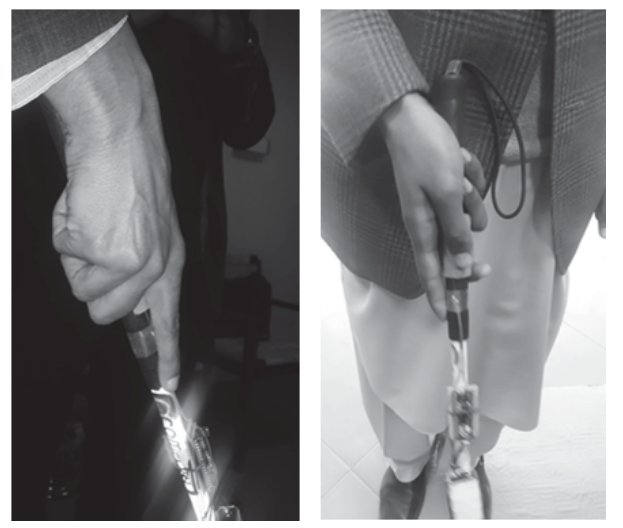

Fig. 4. Smart grip holding with using pointing finger and thumb for sensing
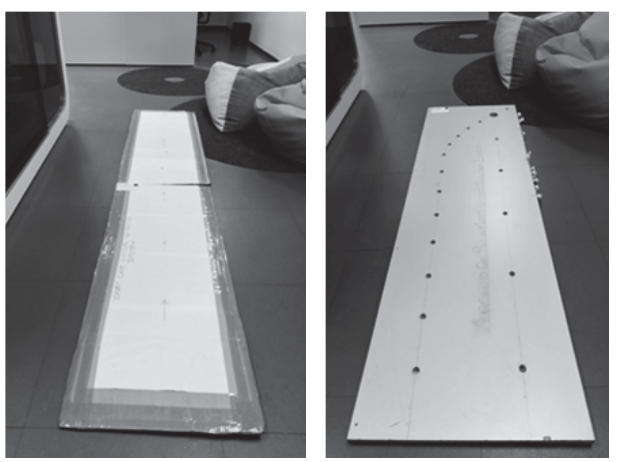

Fig. 5. Sidewalk testbed, finished left side, open with magnet holes right side 
The usability tests were conducted in three cities of Pakistan Lahore, Islamabad, and Abbottabad. The research team consisted of the $1^{\text {st }}$ author of the article and one 35-year old female who has worked in our research group. The duties were distributed like the author conducted the interviews, describe the system and provide assistance if test subjects needed while using the system, and conducted feedback interview. The supporting researcher provided help with logistics and audio/video recording of the interviews and usability tests.

The usability testing was conducted with 14 VIP and blind participants. Their mean age was 32 and median 35 . Participants consisted of two females and twelve males. They were recruited through direct contact to blind associations in Pakistan. The links to the blind association were already established as they were used in earlier studies. An informed consent form was collected from all participants, and they all participated as voluntary basis. Table I describes the test participants by showing categories of visual impairment, gender, and age. (Table I)

TABLE I. DEMOGRAPHICS AND PARTICIPANTS' ACUITY

\begin{tabular}{|c|c|c|c|c|}
\hline $\begin{array}{c}\text { Test } \\
\text { Subject }\end{array}$ & Gender & Age & Visual acuity & On set \\
\hline TS1 & M & 18 & Light perception & By birth \\
\hline TS2 & M & 31 & $20 / 25$ & Late blind, at age 20 \\
\hline TS3 & M & 27 & Light perception & By birth \\
\hline TS4 & M & 38 & Light perception & By birth \\
\hline TS5 & F & 16 & 20/25 & By birth \\
\hline TS6 & F & 33 & Light perception & Late blind, at age of 9 \\
\hline TS7 & M & 26 & Light perception & By birth \\
\hline TS8 & M & 18 & Light perception & Late blind, at age of 9 \\
\hline TS9 & M & 28 & Light perception & By birth \\
\hline TS10 & M & 26 & Light perception & By birth \\
\hline TS11 & M & 30 & Light perception & By birth \\
\hline TS12 & M & 33 & Light perception & $\begin{array}{c}\text { Late blind, } \\
\text { at age of } 15 / 16\end{array}$ \\
\hline TS13 & M & 35 & $\begin{array}{c}\text { Partially blind, } \\
\text { 4-5 m visibility }\end{array}$ & By birth \\
\hline TS14 & M & 39 & Light perception & By birth \\
\hline
\end{tabular}

\section{Experiment setup and testing}

The equipment used in the experiment constitute of the white cane with smart grip and a carboard testbed of a sidewalk on which, a magnetic trail was made using neodymium magnets. Because the testbed has to be carried to different location to reach blind subjects, it comprised of two testbeds, each with 6 feet length. One testbed had magnetic trail in a straight line while second had a curved magnetic trail. When put together, it made magnetic trail on a sidewalk with a turn towards end. The purpose was to test whether VIP or blind test subjects can follow the trail being oriented when a turn comes on sidewalk. An oral introduction of how the system works and how they are supposed to use it was given to all participants. Any question asked by the test participants was answered and they were asked to have a trial navigation on the testbed to familiarize themselves with the system before usability test was conducted.

The usability test scenario was simple. The test subjects had to walk on the testbed, trying to follow the magnetic trail with the help of vibrational cues through smart grip. Each time they detect a magnet, the grip gives vibration feedback as a confirmation for them being on their rout. Then they detect next magnet and keep advancing on the sidewalk being oriented. The success condition for the usability scenario was person successfully detecting and following whole trail from start to end (Fig. 6). We collected feedback from the test subjects by the following questions:

- Can you sense the vibration so that you can use it as navigation aid?

- If such a navigation aid is available, what problem that you face now specifically it could solve?

- How do you feel this would perform if you would use it in a noisy and rush situation?

- If we enhance it with directional guidance using GPS or human guide, and add 3 vibrators parallel to direct right, left, and straight-ahead movements. How that looks?

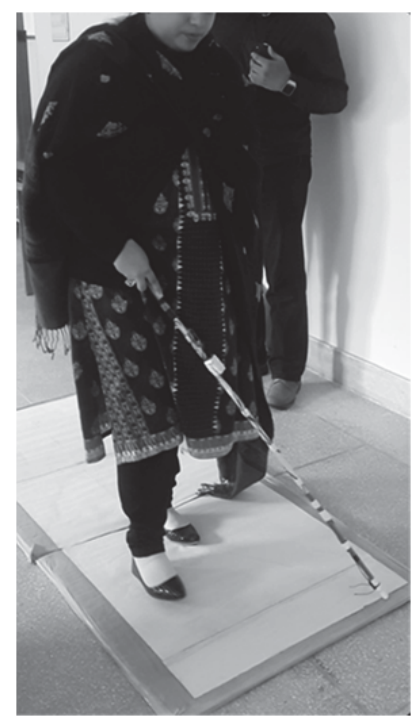

Fig. 6. A test subject walking on the testbed with the help of smart grip

\section{Qualitative study of navigation assistance and environment model}

The qualitative study was conducted to collect information from blind and VIP about navigation assistance and cognitive modeling of the environment of blind and VIP to aid the development of assistive devices. A questionnaire was developed with three main categories; Navigation assistance, Remote navigation assistance, and cognitive modeling of VIP and blind persons' environment. All three categories contain qualitative questions to get deeper understanding of the blind and VIPs feedback. While navigation assistance and remote navigation assistance contain quantitative questions too to find importance of relevance of relating concepts. Five-point Likert scale was used to score the perceived usefulness of assistive tools and ICT devices and burden of caregiving as a Perceived Usefulness Score (PUS), where 1 referring to Nearly always, 2 to Quite Frequently, 3 to Sometimes, 4 to Rarely and 5 to Never.

The participants in this study were the same that in the usability testing. Users feedback of usability testing and 
questionnaire for this study were asked together at the end of usability testing from each test subject. The research team was same in study as well. The author was interviewer while assisting researcher recorded audio of the interview.

\section{E. Environment model: Zone Model}

An environment model was developed as part of this research to aid the development of assistive tools, navigation assistance in this case. The model was named as 'Zone Model'. We divide the blind and VIP's surrounding environment into three zones (Fig. 7).

1) Control Zone: Control zone includes VIPs and blind's home and office environment. These are the places where they can remember the environment and have control over changing something if it is problematic in their navigation. They don't need navigation assistance here as they memorize the environment and have control over change if required.

2) Familiar Zone: The familiar zone is the nearby environment of the VIP and Blind's Control Zone. They can memorize the environment and can have partial control over arranging or changing things if those are problematic for their navigation. This zone includes nearby streets of the house of VIP and blind and corridors of the working place. They need assistance here if the environment change. In this zone, teleguidance assistance that's proposed in this study is a feasible assistance tool. As it do not needs any installment in the environment that may not be possible to install every street in a city.

3) Discovery Zone: The Discovery Zone is the distant environment from VIP or blind Control Zone which is unfamiliar to them and they have no control over changing it. They need navigation assistance in this zone. In this zone, trail-based assistance that's proposed in earlier study and teleguidance assistance that's proposed in this study are feasible support tool. The trail-based assistance is $1^{\text {st }}$ resort as this infrastructure can be installed and taken care in the city centers for example. While, if required teleguidance assistance is the $2^{\text {nd }}$ line of resort to get assistance in this zone.

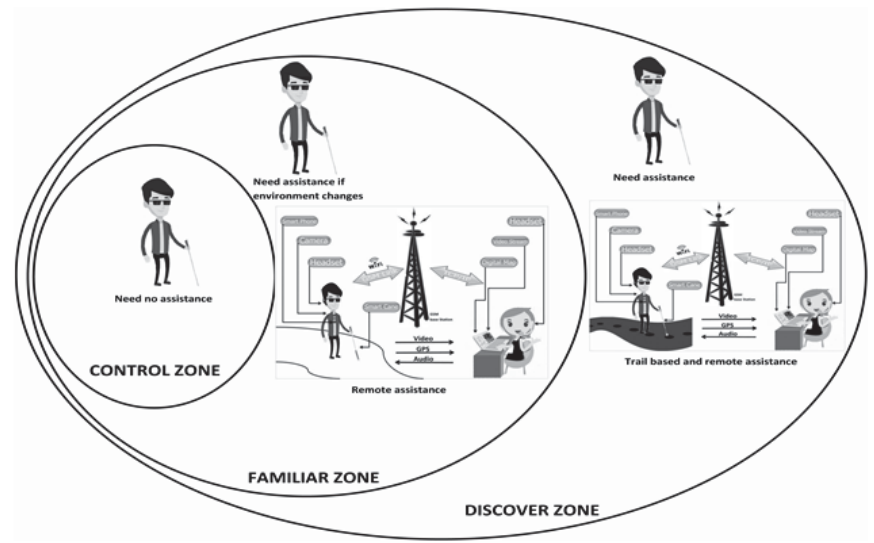

Fig. 7 An environment model with three zones and what navigation assistance is appropriate in each zone

\section{RESULTS}

This chapter presents the findings from the usability test and qualitative study on navigation assistance and environment.

\section{A. Usability feedback after testing}

In the usability test, we asked test subjects to focus on vibration and comment if they can sense the vibration so that they can use it as navigation aid. All participants argued that they can sense it and follow the guidance. One participant said: "It is like paving block, but interactive". Test subjects thought that it is helpful in navigation and one participant commented that it can be especially helpful on turns. Two users gave feedback about intensity and suggested that it could be a bit more intensive and not so slow. One test subject wondered that even though it would be helpful in navigation, there will be some learning curve and it can take some time to familiarize with it. One participant commented that this type of system could be very cost-effective especially in region of South Asian.

We asked also what current problems our system could solve. Participants gave several examples what kind of problems the system could solve:

- Avoiding the cane get stuck

- Avoiding falling when changes on the path

- Help to guide in turnings

- Help to orientate

- Help to follow the path even the sidewalk ends or there are broken parts

- Help to navigate in unfamiliar environments

- Enabling concentrating on something else while following the guidance from the interactive systems

- Following guidance trough vibration even in the noisy environment

- No need to ask help for navigation so often

One participant commented: "Basic challenge of white cane is it's a dumb device while this [system] is interactive. So it give more confidence and you feel good. Even though we know white cane guides us well but at time like if there is noise, that makes it hard to follow white cane well. As that effects our echolocation capability that we use while using white. So that effects white cane sensing." (TS12)

we also asked users opinion about the usage of the system in a noisy and rush situation. Many participants commented that the system could be adjustable, and the vibration could be stronger as one participant (TS6) commented: "I think it should be adjustable. Like when I am at relatively quiet place, I put it at low intensity. But if I end up at noisy place, I can increase it. Another participant (TS8) said: "I heard about some canes that gives oral feedback, this [system] is better I think because I can miss what it said if crowd or noise, but vibration is what my hand will feel.". Some participants commented that it would be easier to notice vibration if its intensity is increased a bit. One test subject (TS14) commented: "I think a customized setup that everyone adjusts to his ease and one can change according to environment will be appropriate. Just like mob apps." 
We were also interested in hearing participants opinions of new features. We asked what they would think about the system could guide using GPS or human guide. In addition, we asked, would it good to add three parallel vibrators to direct right, left, and straight ahead. Participants opinions to new features varied. Approximately half liked the idea and other half thought that adding more vibrators can be confusing. One participant (TS1) thought that: "if only vibrators will vibrate, there is no confusion. But if whole grip vibrates like it does now, it can create confusion."

This type of situation could be avoided by putting vibrators a bit far from each other for instance, right one under fingers, left one under palm of the hand, and middle under thumb.

Another user (TS2) also said: "It is possible to get confused if there are more than one vibrator installed side by side. The solution to this can be when there comes a decision point, either all three vibrators vibrate at the same time to let me know a decision point has arrived OR a beep can also serve same purpose".

One participant (TS11) commented that it can be confusing in the beginning but with practice it could be easier. He gave an example what he would like to have in the system: "What I would like to add is that one of our problem is road crossing. So, if something that helps us in that is added to it or such system will be very useful".

\section{B. Attitudes towards navigation assistance}

This section presents the questionnaire results relating to attitudes and opinions towards navigation assistance (Table II). The answers to statements can be divided to following aspects:

1) Mobility and independence $(Q 1,4,5,6,8,16)$ : Participants though that navigation assistance makes going outdoors more pleasant (1.6) and they need NA quite frequently (2.0) Participants attitudes towards NA is very positive, because they though that they are willing to change their daily routines for getting a new kind of NA (1.6). (Table II)

2) User experience and usability (Q2, 7, 9, 17, 19): Participants had postivite attutudes about the usage of the NA. They thought that NA is easy to use (1.6) and they did not feel nervous when using NA (4.1) (Table II)

3) Self-confidence and safety $(Q 10,15,18,20)$ : Participants perveiced that sometimes (2.9) technological NA is somewhat intimidating. However, participants though that they have sufficient knowledge to use NA (1.7) and those are easy to learn (1.6). Participants preceived that NAs make them feel safe (1.6)

4) Social aspects $(Q 11,12,13,14,24,25)$ : Participants perceived that people supports them to use NAs (Q11: 2.1 and Q14: 2 and Q13: 3). They also think quite frequently (2) that it is good that people close to hem/her know their location. Participants think that people close to them are willing to change their daily routines, if they receive a new kind of NA (1.9). Participants do not find it annoying when surrounding people notice that they are visually impaired (3.6). (Table II)

5) Expenses, time and effort $(Q 3,21,22,23)$
According to questionnaire, participants are willing to spent money to buy a new NA (1.4) and also spend their time to learn to use them (1.6). They also think that NAs are reasonably priced (2.1). (Table II)

TABLE II. AtTitudes towards NAVIGATION Assistance (NA). A MEANING OF THE SCALE RESULTS (R): 1 = NEARLY ALWAYS, 2 = QUITE FREQUENTLY, 3 = SOMETIMES, $4=$ RARELY, $5=$ NEVER

\begin{tabular}{|c|c|c|}
\hline $\mathbf{Q}$ & Likert Scale arguments & $\mathbf{R}$ \\
\hline \multicolumn{3}{|c|}{ 1) Mobility and independence } \\
\hline 1 & NA makes going outdoors more pleasant. & 1.6 \\
\hline 4 & Using NA has become a habit to me. & 2.1 \\
\hline 5 & I need NA every time I go outdoors. & 2.0 \\
\hline 6 & In my opinion NA is useful when I am outdoors. & 1.7 \\
\hline 8 & If I use NA, I will increase my chances to get where I want. & \\
\hline 16 & If I have a problem with NA, I know from whom I ask help. & 1.8 \\
\hline \multicolumn{3}{|c|}{ 2) User experience and usability } \\
\hline 2 & I like using NA. & 1.6 \\
\hline 7 & NA increases the speed of doing everyday activities. & 1.9 \\
\hline 9 & I find NA tools easy to use. & 1.6 \\
\hline 17 & I feel nervous using NA. & 4.1 \\
\hline 19 & Using NA is a good idea. & 1.6 \\
\hline \multicolumn{3}{|c|}{ 3) Self-confidence and safety } \\
\hline 10 & Learning to use NA tools is easy for me. & 1.6 \\
\hline 15 & My knowledge of using NA is sufficient. & 1.7 \\
\hline 18 & Technological NA is somewhat intimidating. & 2.9 \\
\hline 20 & NA makes me feel safe. & 1.6 \\
\hline \multicolumn{3}{|c|}{ 4) Social aspects } \\
\hline 11 & $\begin{array}{l}\text { People, who are important to me, think that I should use } \\
\text { NA. }\end{array}$ & 2.1 \\
\hline 12 & $\begin{array}{l}\text { I find it annoying when surrounding people notice I am } \\
\text { visually impaired. }\end{array}$ & 3.6 \\
\hline 13 & The society has been supportive in the use of assistive tools & 3.0 \\
\hline 14 & $\begin{array}{l}\text { Instructors or medical staff has been supportive in the use of } \\
\text { assistive }\end{array}$ & 2.0 \\
\hline 24 & $\begin{array}{l}\text { I think that people close to me are willing to change their } \\
\text { daily routines, if I receive a new kind of NA. }\end{array}$ & 1.9 \\
\hline \multirow[t]{2}{*}{25} & It is good that people close to me know my location. & 2.0 \\
\hline & 5) Expenses, time and effort & \\
\hline 3 & NA tools are reasonably priced. & 2.1 \\
\hline 21 & I am willing to spend money in order to buy new NA. & 1.4 \\
\hline 22 & $\begin{array}{l}\text { I am willing to spend my time in order to learn to use new } \\
\text { NA. }\end{array}$ & 1.6 \\
\hline 23 & $\begin{array}{l}\text { I am willing to change my daily routines, if I receive a new } \\
\text { kind of NA. }\end{array}$ & 1.6 \\
\hline
\end{tabular}

\section{Opinions relating to distance and environment aspects}

In the questionnaire we asked four questions relating to distance aspects: 1. I would like to select when people close to me know my location, 2. Is it easy to get assistance when needed?, 3. Do you ever used mobile to ask for assistance if you were in need of assistance? and 4. Would you accept help from an unknown person if it is recommended by someone you trust?

Almost all (13/14) participants would like to select when people close to them could see their location (Fig. 8). Only one test subject argued that it is easy to get assistance when needed. 11 of the participants thought that it is somewhat easy and for two participants it is not easy. Ten test subjects said that they use mobile phone for asking assistance. Also, ten participants commented that they can accept help from some unknown person if some trusted person has recommended that him or her. 
Relating to environment aspects we asked: 1. What/when are the places you feel you are safe/in control of situation/life? and 2. If there is an area out of these where you feel safe and in-control, How and why you feel? All participants said that they feel safety at home. Then participants though that they feel safe also in school or workplace environment. One test subject feels safe anywhere at daytime and one when he is in places, where accessibility is easy. Two persons answered that when visiting relatives' or wife's home. Six participants though this feeling has increased over last 3-5 years. Seven felt that it has not changed, and one commented that feeling of safety has decreased.

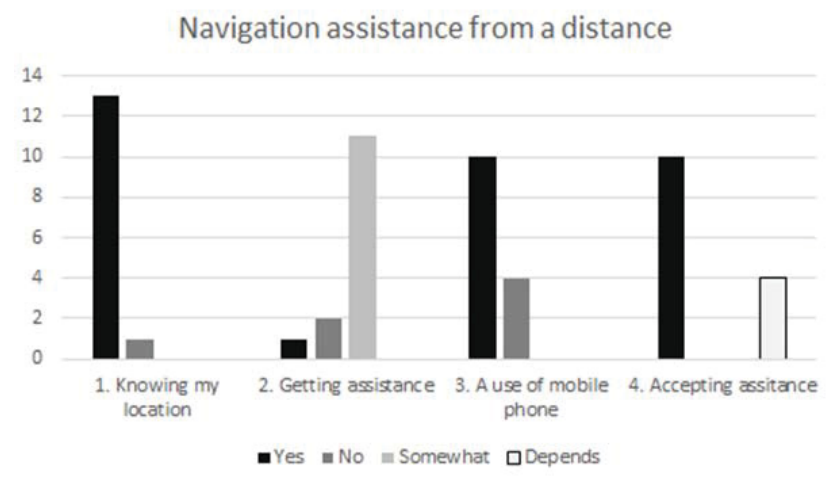

Fig.8 Participants attitudes towards navigation assistance from a distance

When we asked from participants how often they visit unfamiliar places, seven of them answered that sometimes and two very rarely. Five participants said that they do not go (independently) to unfamiliar places. According to questionnaire, when participants go unfamiliar places they need to prepare beforehand. Test subjects answered that they need to collect surrounding information (9), transportation or path information (6) and weather information (3). Five participants do not go unfamiliar places and therefore not need to prepare in that sense. Seven of participants call to someone if they need assistance. Participants gave different reasons, why they need to go unfamiliar places: Meet friends or someone (9), go shopping (1) and go to work sometimes (1).

We also asked how anxious they are at unfamiliar places. Three answered that they are somewhat and two not much or at all anxious. Three persons answered that they are sometime anxious. One participant said that yes when the weather is bad.

In the questionnaire, we asked how far from home participants feel safe. Only three answered that they feel safe everywhere and one said that most of the places in daytime. Two felt safe in familiar place. Two said that they feel safe at home and five also near to home. When we asked how often and how far participants go from home without planning, only there answered that sometimes and three that rarely. Half of the participants said that they do not go out without planning. Those who go outside, said that they go quite near or about 10.15 kilometers far from home. Participants estimated that when they go out without planning they stay there about half an hour or one hour or it depends on the situation. Half of the participants prefer to outside without planning during the daytime. One participant said that in the morning or daytime.
In the questionnaire we were also interested in to know what kind of things participants may be afraid when they are at home, in school/ work or far away from these familiar places. Participants mentioned many different things: (wild or fierce) animals (10) including e.g. cats, dogs, birds, livestock; obstacles/insects (5); earthquakes (4); traffic (3), falling down (2); electricity/appliances (2), bad weather (1). Only three participants though that they are not afraid at all.

\section{SURVEY FOR FAMILY MEMBERS AND CARETAKERS}

Assistive technology is used by person with disabilities to increase, maintain, or improve their functioning of everyday life activities. The assistive technology can be available in the form of a service, device, application, or tool that helps disables people and/or their caretakers to maintain functioning capabilities of the persons being taken care or assisted. Assistive technology can come in the form of mechanical or electrical devices, wirelessly networked sensors (WSN), IoT applications, cloud, or edge-based devices. Despite what class of assistance those belong to, assistive devices are found to have capabilities to improve users' functioning, increase participation, and reduce functional decline.

On caretaker's benefit, assistive technology has been found to reduce the burden of caretaking. The prime benefit ICT based assistive technology brings to caretakers is decrease of demand related to care and monitoring of the assistance recipient. In the case of ICT based assistance, the additional benefit than traditional assistive tools is that a person can be assisted or contacted from a distance, not being physically present with care recipient. This car ne especially critical in the incident of an emergency or need of assistance at an unexpected hour. Caretaker, whether family members, friends, or volunteers are a valuable resource of taking care of disabled people with their unpaid contribution.

ICT and IoT based assistive tools are going to be of a lot value for disabled persons and their caretakers with the value the brought into performing and receiving assistance. This study was designed with the aim of examining to what extent the caretakers of VIP and blind utilize ICT-based assistive technologies in their caregiving activity, and what are the needs for and perceived usefulness of ICT based assistive technologies.

\section{A. Materials and methods}

A questionnaire was developed to get feedback from caretakers of VIP and blind people. The questionnaire consisted of five main categories: Background, Assistive technologies and tools, Time utilization, Burden, and Coping. The questionnaire contained both qualitative and quantitative questions. Qualitative study was used for questions about assistive tools and ICT devices to collect information about the needs and expectations assigned to the technology, and about time utilization to collect information about remote and onsite assistance. Five-point Likert Scale was used to score the perceived usefulness of assistive tools and ICT devices and burden of caregiving as a Perceived Usefulness Score (PUS). 


\section{B. Participants}

The participants were contacted through third party blind welfare organizations in Pakistan. The contacts to those organization were already established as part of ongoing user study for this research project. Two caretakers, one sighted wife of a blind test subject who participated in VIP study (reported in chapter IV) in the city of Lahore city and the $2^{\text {nd }}$ caretaker a volunteer person who takes care of his 7 friends and acquaintances after working hours in Abbottabad city were interviewed. Few of the persons taken care by him participated in the above reported VIP study as well.

Caretaker 1 (CT1) has a nine years' experience of assisting and Caretaker 2 (CT2) had assisted 2,5 years. CT1 lived in a walking distance from the person that he assisted and CT2 lived in the same household, because she assists her husband.

\section{B. Findings}

Both caretakers used ICT, mostly mobile phone by calling for assisting from a distance and they did not use other ITs while assisting. Caretakers did not have any experience with telemonitoring assistance (e.g. door alarm, GPS tracking device, fall detector), but both had willingness to use telemonitoring or remote assisting system.

Participants said that the most common tool or device they used for assisting is mobile phone and they thought that it helps them sometimes (CT1) or quite frequently (CT2). The most common assistive tool their assist was using was white cane. CT2 mentioned also mobile phone.

Caretakers hoped that by new navigation assistance system they could save time for their selves (CT1) and that they could help more through mobile phone (CT2).

\section{ACCEPTANCE STUDY WITH VIP AND BLIND PERSONS}

The usage level of assistive tools by VIP and blind people varies for instance, by geography, age, and visual acuity. Prior studies reveal that usage of tools percentage is higher within full blind as compared to partially blind people, $26 \%$ and $6 \%$ respectively. Reasons for technological assistance not adopted widely by VIP and blind people include but not limited to being expensive, not widely available, no knowledge and limitation of technical support and training. As the aim of this research was to develop novel ICT based navigation assistance for blind and VIP, it was found worthwhile to conduct a study to find degree of acceptance, acceptance issues, and willingness of blind and VIP to accept kind of assistances which development was aim of this research.

\section{A. Selection of suitable technology acceptance model}

One of the planning issues to conduct this study was to choose an appropriate acceptance model that examines acceptance of technology by consumers, blind and VIP in this case. Several acceptance models are popular amongst research community and are being used, e.g. the Technology Acceptance Model (TAM), the Motivation Model (MM), the Theory of Planned Behavior (TPB) to enlist a few. Venkatesh et al. designed the Unified Theory of Acceptance and Use of
Technology (UTAUT) by incorporating eight IT acceptance models. Later Venkatesh et al. [29] made some modifications in the model. The UTAUT2 model can be used by organizations to gain knowledge on improving the design and marketing of their consumer product [30]. This altogether made the UTAUT2 a suitable choice of acceptance model to be used in this study to find out acceptance issues in technological assistive tools for VIP.

The questions set was modified to suit the VIP context. The UTAUT2 model constructs are performance expectancy (PE), effort expectancy (EE), social influence (SI), facilitating conditions (FC), hedonic motivation (HM), price value (PV) and habit (HA) which influence behavioral intention to use a technology and usage behaviors. The constructs are moderated by individual differences such as age and gender which are asked as background questions (BG). As the modified questionnaire for this study was meant for assistive tools and for special user groups, it was felt that some questions from the first version of the UTAUT model should be included, especially from group of anxiety (ANX) and attitude (ATT), which had been dropped from the UTAUT2 model.

In addition, groups of questions not related to technology acceptance were included. Those include questions assessing the potential for specific assistive technologies for the purposes of developing prototypes (SPE). The prototypes include teleguidance system with location finding functionality. Also questions on willingness to adapt new technologies (TA) by VIP and previous experience (PEx) of using navigation assistance, support persons (SP) availability, habits, willingness to change routines or practices (RO), and whether there are independency and privacy issues (IP), were included. With these questions, new information for developing prototypes was hoped to receive.

\section{B. Participants}

The participants were recruited through three third sector associates in the city of Oulu region. The research was presented to them and volunteers were recruited. The location of the interview was based on the preference of the interviewees being either at their own residence, at the office of the Northern Ostrobothnia Association for the Visually Impaired or at the University of Oulu, or if they preferred, any given public location was offered. Most of the participants chose their home location, while three chose University as preferred location for the interview and four participants were interviewed at the office of the Northern Ostrobothnia Association for the Visually Impaired.

The study was conducted with 19 participants, whose age varied from 19 years to 82 years and in the analysis they are divided to three age groups: 18-30, 40-63 and 64-99 years old. Fig. 9 shows the percentage of participants' distribution by gender and age groups. Within the youngest and oldest age group, women seemed to be more interested in participating in this study while men dominate the middle age (Fig 9). The level of vision varied from total blindness to low vision. Fig 10 illustrates that there were more VIP who have lost their vision gradually as been born VIP. 
Approximately half of the participants were smartphone users. The eldest age group was using least of navigation assistance. Fig 11 shows how the different age groups own and use mobile devices. The youngest age group is most keen on using mobile devices in contras of the eldest who are not that interested using them.

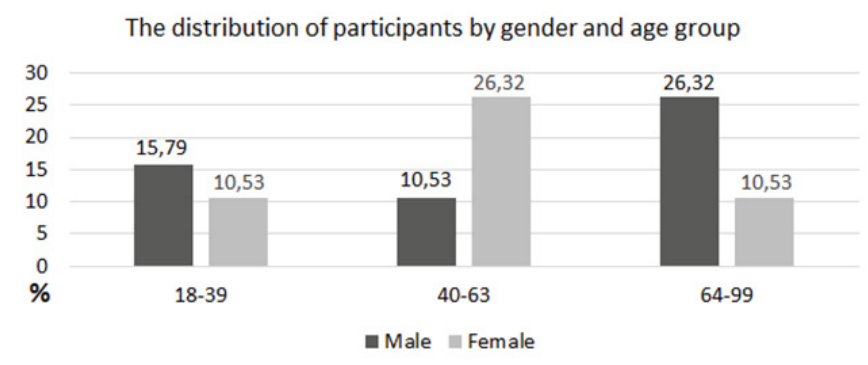

Fig. 9. Demographics

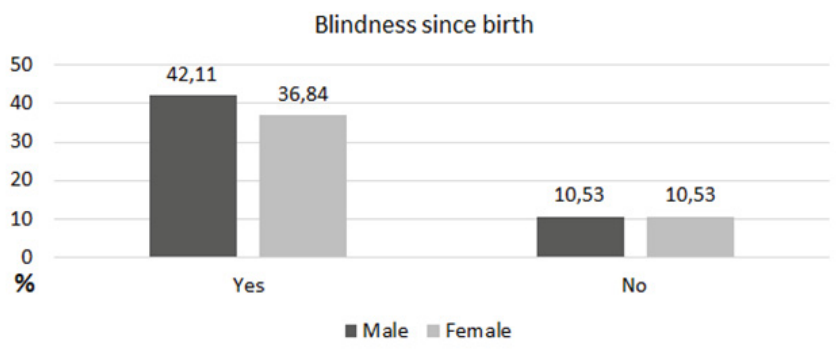

Fig. 10. Percentage of participant's blindness since birth

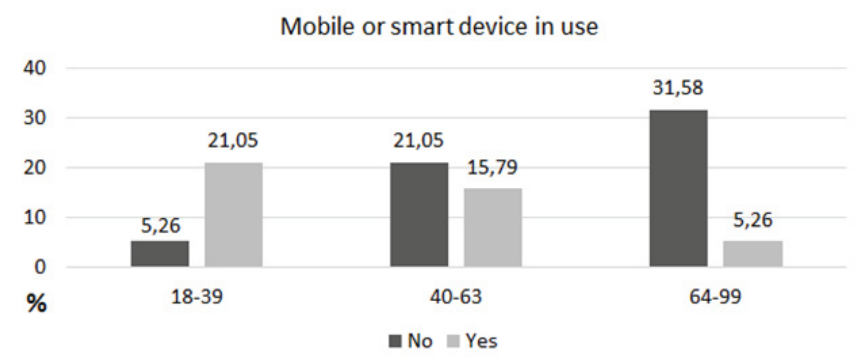

Fig. 11. Percentage of mobile or smart device users by different age group

\section{The questionnaire and data collection}

The questionnaire was divided into two parts. the first part was a semi-structured interview set consisting of 10 questions (question numbers 1-10). There was a likely possibility of asking few possible follow-up questions. The second part of the questionnaire consists of 26 statements which are to be answered with a 5-point Likert scale (question numbers 11-36).

\section{Data processing}

Both numeric and narrative data were collected. Quantitative data was analyzed first, that provided a good background for qualitative analysis. Though as number of participants was relatively low (19), the statistical results are directive. The data was analyzed with regression test, variance tests, and non-parametric tests. The quantitative statistical tests were done with 3 background variables: gender, age, and age group. Regression analysis is meant to find out linear dependency of two variables. Variables included were age, gender, age group and UTAUT2-model constructs. Crosstabulation was used to find connection between two variables. The connections were investigated between attitude and gender, age or age group. Variance analysis was done on the material. As data set was small, factor analysis that's is meant for describing same variance phenomenon on fewer dimensions: In other words, to compress the information. This would make the interpretation of the factors easier.

As the questionnaire served mainly the quantitative aspect of the research, the quantity of qualitative data collected was not huge. The qualitative analysis of the data was done in two phases. In the first phase, the questions were selected based on how they may give answers to the selected topics. For example, questions $6,8,31,32,35$, and 36 were chosen to find out responses for "Technological adaption". In the second phase, it was checked whether any data is missing to answer any topic. Use of a simple keyword list was thought at first but it was not used as amount of data was not huge. Each interview was transcribed and data containing answers to different categories were easily found without using it.

\section{E. Results}

The data set collected through modified UTAUT2 - model questionnaire was used to measure if UTAUT2 acceptance model is usable when assistive technology for VIP is studied. The questionnaire developed was a measure to find UTAUT2model variables. The factors can be derived from the answers If the questions successfully measure each variable. Factors can then be analyzed further. When measured, ff the internal consistency among the answers is high, it indicates that the model is suitable for this kind of study. In vice versa, it is a sign that the questions do not measure UTAUT2 model variables correctly.

The Varimax-Rotation method was used for factoring the modified UTAUT2-model variables. The internal consistency has been checked for each variable. Further tests were done for each variable included in a factor and which support the UTAUT2 model. Factors are scrutinized by factor loadings, commonalities and Cronbach's alpha.

Fig. 12 shows the results of the analysis. It shows that UTAUT2-model is usable for studying acceptance issues by VIP and blind people. In the figure, middle row presents the UTAUT2 constructs and bottom row presents the questions which measure these constructs. UTAUT2-model variable "Social Influence" is measured by three questions. The analyzed results indicate that these questions do not measure the variable, because the three questions and answers do not load on the same factors and their internal consistency is low: The Cronbach's alpha is negative $(-0.31)$. This means that this data do not support that UTAUT2-model variable. The same goes with construct "Facilitating Conditions" with low Cronbach's alpha (0.11) showing there is no internal consistency between the variables. Other variables than the mentioned two support the UTAUT2-model when scrutinizing Cronbach's alpha value. The verdict for whether the data supports the construct is received not only by Cronbach's alpha 
value but by commonality and factor loadings as seen on Table III. Facilitating Conditions has a low commonality score. Other variables than the mentioned two support the UTAUT2-model when scrutinizing Cronbach's alpha value.

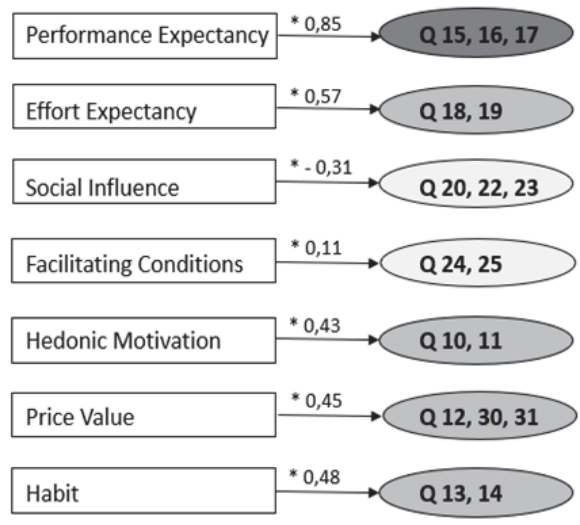

Fig. 12. Factor analyze by using UTAUT2 constructs (loadings: $0,61-0,85$ )

As a result, it seems that the model is usable for this kind of research. Nevertheless, some questions don not support or measure given variable. Some questions should be considered and tested again. Consideration here may mean that the Finnish translation of the questions could be adjusted. Or maybe, in retrospect, the removing few of the UTAUT2 questionnaire statements aside could have been an error. This was done to shorten the interview duration. Also, some of the UTAUT2 questions were hard to modify to suit for this special purpose, the assistive navigation tools for VIP. As a conclusion for quantitative analysis, it can be said that men and women react differently for technological assistance tools. Women do have more negative attitudes towards technology than men (Fig. 13). While this is not much evident in age groups (Fig. 14).

TABLE III.

THE ANALYZED UTAUT2 CONSTRUCTS

\begin{tabular}{|c|c|c|c|}
\hline $\begin{array}{c}\text { Question } \\
\text { number }\end{array}$ & Commonality & Loadings & $\begin{array}{c}\text { Cronbach's } \\
\text { Alpha }\end{array}$ \\
\hline \multicolumn{5}{|c|}{ Performance Expectancy } \\
\hline 15 & 0.77 & 0.88 & 0.85 \\
\hline 16 & 0.80 & 0.90 & - \\
\hline 17 & 0.85 & 0.92 & - \\
\hline \multicolumn{5}{|c|}{ Effort Expectancy } \\
\hline 18 & 0.70 & 0.83 & 0.57 \\
\hline 19 & 0.70 & 0.83 & - \\
\hline \multicolumn{5}{|c|}{ Social Influence } \\
\hline 20 & 0.94 & - & -0.31 \\
\hline 22 & 0.76 & 0.87 & - \\
\hline 23 & 0.80 & -0.77 & - \\
\hline \multicolumn{5}{|c|}{ Facilitating Conditions } \\
\hline 24 & 0.55 & 0.74 & - \\
\hline 25 & 0.55 & 0.74 & - \\
\hline \multicolumn{5}{|c|}{ Hedonic Motivation } \\
\hline 10 & 0.65 & 0.80 & 0.43 \\
\hline 11 & 0.65 & 0.80 & - \\
\hline \multicolumn{5}{|c|}{ Price Value } \\
\hline 12 & 0.37 & 0.82 & 0.45 \\
\hline 30 & 0.42 & 0.65 & - \\
\hline 31 & 0.66 & 0.61 & - \\
\hline \multicolumn{5}{|c|}{ Habit } \\
\hline 13 & 0.72 & 0.84 & -88 \\
\hline
\end{tabular}

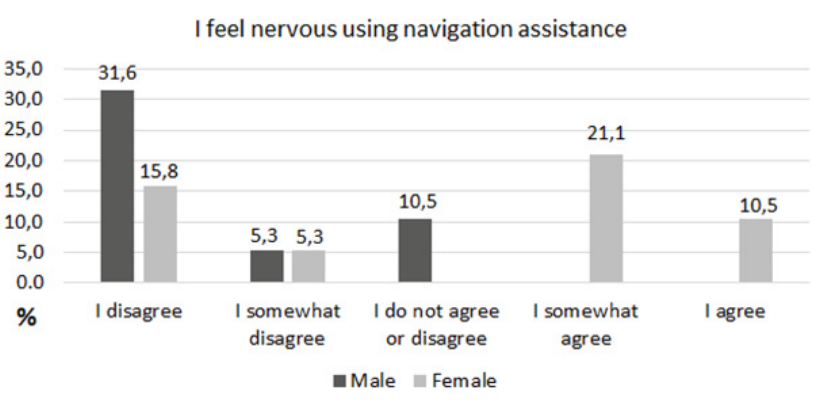

Fig. 13. Nervousness towards navigation assistance by gender

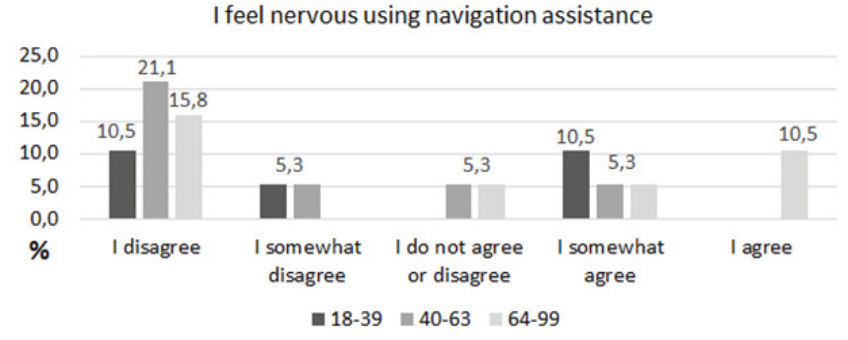

Fig. 14. Nervousness towards navigation assistance by age group

\section{DISCUSSION}

In this paper, we have introduced the tele-guidance based navigation assistance system to assist blind and VIP in navigation. In addition, we have presented different user studies that we have conducted in Pakistan and Finland. In Pakistan we conducted usability study and qualitative study where we had 14 blind and VIP and two caretakers. In Finland we conducted acceptance study with 19 blind and VIP. We acknowledge that our sample is relatively small, and we cannot generalize our results. Especially, we should involve more caretakers in the future. It is very important understand the cooperation and assisting needs between blind and VIP with their caretakers while using co-operative navigation assistance. In addition, we acknowledge that our findings are found from studies which are conducted only on two countries and some of the findings can be culture and society specific. Therefore, it would be useful to study the tele-guidance based system also in other countries. However, we regard sharing these findings as useful and important in order to try to improve and developed new navigation assistance systems for blind, VIP and their caretakers.

\section{CONCLUSION}

In this paper we present a tele-guidance based navigation assistance system to assist blind and VIP in navigation. The approach of the system is based on the idea that a blind pedestrian can be assisted by spoken instructions from a remote caretaker who receives a video stream from a camera carried by the visually impaired user. Different studies of usability testing, environment, and acceptance of the assistive technology were 
described. The scenarios for the testing of overall system for both VIP and remote caretaker to evaluate the usability factors of the system in each of its testing phases were specified. We expected that participants could complete their tasks with the help of the proposed system.

A qualitative study using a modified questionnaire based on Unified Theory of Acceptance and use of Technology (UTAUT2) to study attitudes of VIP towards navigation tools (technological \& non- technological) was reported in this paper. As a conclusion for the qualitative study, it can be said that VIP in general have an interest towards technology and new kind of assistive tools. Unfortunately, the participants have not found much navigation assistance devices suitable for them. This means that the VIP should be taken into design process. Technology may increase the acceptance in the future, but so far it has not been the case. Furthermore, every-day mobile technology is already becoming common among VIP's and applications for those devices can be designed. Advantages of mobile applications include non-stigmatization and affordability. Many of the VIP are used to white canes, and also for the cane's extra function as the signal cane. Therefore, creating light weight, low-price extra-functions for the cane is a good idea. Especially there is a need to encourage elderly VIP to acquire and use assistive tools.

\section{ACKNOWLEDGMENT}

The first author has received funding for his $\mathrm{PhD}$ research from the OASIS Research Unit and the Scholarship Fund of the University of Oulu. Also, UniOGS Travel grant is awarded for studies. We want to thank all collaborations and participants for their invaluable effort and feedback during this research.

\section{REFERENCES}

[1] S. Bhatlawande, M. Mahadevappa, J. Mukherjee, M. Biswas, D. Das and S. Gupta (2014). Design, development, and clinical evaluation of the electronic mobility cane for vision rehabilitation. IEEE Transactions on Neural Systems and Rehabilitation Engineering, 22(6), 2014, pp. 1148-1159.

[2] Global data on visual impairments 2010 http://www.who.int/blindness/GLOBALDATAFINALforweb.pdf?ua $=1$

[3] D. Pascolini and SP. Mariotti. "Global estimates of visual impairment: 2010." British Journal Ophthalmology Online First published December 1, 2011 as 10.1136/bjophthalmol-2011-300539.

[4] R.R. Bourne, S.R. Flaxman, T. Braithwaite, M.V. Cicinelli, A. Das, J.B. Jonas, ... and K. Naidoo. Magnitude, temporal trends, and projections of the global prevalence of blindness and distance and near vision impairment: a systematic review and meta-analysis. The Lancet Global Health, 5(9) x2017. e888

[5] N. Bourbakis. Sensing Surrounding 3-D Space for Navigation of the Blind, IEEE Engineering in Medicine and Biology Magazine, Jan/Feb, 2008, pp. 49-55.

[6] A. G. Nicholas, and G. E. Legge, "Blind navigation and the role of technology," in The Engineering Handbook of Smart Technology for Aging, Disability, and Indepen- dence, 2008, pp. 479-500.

[7] A. Riazi, F. Riazi, R. Yoosfi, and F. Bahmeei, "Outdoor difficulties experienced by a group of visually impaired iranian people," J. Curr. Ophthalmol., Vol. 28, no. 2, pp. 85-90, 2016.

[8] R. Manduchi, S. Kurniawan, and H. Bagherinia, "Blind guidance using mobile computer vision: a usability study," in ASSETS, 2010, pp. 241-242.
[9] B.-S. Lin, C.-C. Lee, and P.-Y. Chiang, "Simple smartphone-based guiding system for visually impaired people," Sensors, Vol. 17, no. 6, 2017, pp. 1371.

[10] Y. Zhao, E. Kupferstein, D. Tal, and S. Azenkot, "It looks beautiful but scary: How low vision people navi-gate stairs and other surface level changes," in Proceedings of the 20th International ACM SIGAC-CESS Confer- ence on Computers and Accessibility, ACM, 2018, pp. 307-320.

[11] A. Bhowmick, and S. M. Hazarika, "An insight into assistive technology for the visually impaired and blind people: state-of-the-art and future trends," J. Multi- modal User Interfaces, Vol. 11, no. 2, pp. 149-172, 2017.

[12] B. Nordqvist, B. Nähdä. In A-L. Salminen (Eds.), Apuvälinekirja, 2003, pp. 94-110. Helsinki: Kehitysvammaliitto, Oppimateriaalikeskus.

[13] B. Ravneberg. "Identity politics by design: users, markets and public service provision for assistive technology in Norway". Scandinavian Journal of Disability Research vol. 11, No. 2, 2009, pp. 101-115

[14] A.-L. Salminen. Apuväline toimintaa edistämässä. In A-L. Salminen (Eds.), Apuvälinekirja, 2003, pp. 18-32. Helsinki: Kehitysvammaliitto, Oppimateriaalikeskus.

[15] C.D. Wickens, J.G. Hollands, S. Banbury and R. Parasuraman. Engineering psychology \& human performance. Psychology Press. 2015.

[16] S. Söderström and B. Ytterhus. "The use and non-use of assistive technologies from the world of information and communication technology by visually impaired young people: a walk on the tightrope of peer inclusion". Disability \& Society vol. 25, issue 3. 2010, pp. 303-315.

[17] P. Strumillo. Electronic Systems Aiding Spatial Orientation and Mobility of the Visually Impaired. In: Hippe Z.S., Kulikowski J.L., Mroczek T. (eds) Human - Computer Systems Interaction: Backgrounds and Applications 2. Advances in Intelligent and Soft Computing, vol 98. 2012. Springer, Berlin, Heidelberg. https://doi.org/10.1007/978-3-642-23187-2 24

[18] V. Garaj, Z. Hunaiti and W. Balachandran. "Using remote vision: the effects of video image frame rate on visual object recognition performance". Systems, Man and Cybernetics, Part A: Systems and Humans, IEEE Transactions on, 40(4), 2010, pp. 698-707.

[19] V. Garaj, R. Jirawimut, P. Ptasinski, F. Cecelja and W. Balachandran: "A system for remote sighted guidance of visually impaired pedestrians", British Journal of Visual Impairment,vol. 21, 2003, pp. 55-63.

[20] M, Bujacz, P. Baranski, M. Moranski, P. Strumillo and A. Materka. Remote mobility and navigation aid for the visually disabled. In Proc. 7th Intl Conf. on Disability, Virtual Reality and Assoc. Technologies with Art ArtAbilitation, in PM Sharkey, P. Lopesdos-Santos, PL Weiss \& AL Brooks (Eds.), 2008, pp. 263-270.

[21] P. Baranski, M. Polanczyk, and P. Strumillo. A remote guidance system for the blind. In Proc. IEEE Int. Conf. on e-Health Networking Applications and Services, 2010, pp. 386-390.

[22] API AI Co. Retrieved 30th Sep 2016: http://www.api-kk.com/denshihakujo/

[23] BlindMaps.org. Retrieved 30th Sep 2016: http://www.blindmaps.org. 2016.

[24] Unity 3D. Retrieved 30th Sep 2016: https://unity3d.com/

[25] Linphone. Retrieved 30th Sep 2016: http://www.linphone.org/

[26] ZRTP. Retrieved 30th Sep 2016: http://tools.ietf.org/html/draftzimmermann-avt-zrtp

[27] Answers to your ZRTP Questions. (2016) Retrieved 30th Sep 2016: https://www.silentcircle.com/products-and-solutions/technology/zrtp/

[28] B. Chaudary and P. Pulli. "Smart Cane Outdoor Navigation System for Visually Impaired Deaf-blind and Blind Persons". Commun Disord Deaf Stud Hearing Aids 2014, 2: 125. doi:10.4172/23754427.1000125

[29] V. Venkatesh, M.G. Morris, G.B. Davis, and F.D. Davis, "User acceptance of information technology: Toward a unified view". MIS Quarterly vol 27, No. 3, 2003, pp. 425-478.

[30] Venkatesh, V., Thong, J.Y.L., and Xu, X. "Consumer acceptance and use of information technology: Extending the unified theory of acceptance and use of technology". MIS Quarterly vol 36. No 1. 2012, pp. 157-178. 